\title{
Ambulatory blood pressure monitoring in clinical trials with antihypertensive agents
}

\author{
Anton $\mathrm{H}$. van den Meiracker * \\ Department of Internal Medicine I, University Hospital Dijkzigt, Dr. Molewaterplein 40, 3015 GD Rotterdam, Netherlands
}

Received 18 March 1994; revision received 24 May 1994; accepted 27 May 1994

\begin{abstract}
Ambulatory blood pressure monitoring (ABPM) is being used increasingly for the evaluation of antihypertensive agents in clinical trials. In this brief review several aspects of ABPM are discussed. In particular, attention is paid to the extent to which ABPM is subject to a placebo response and the extent to which the sample size of the study population can be reduced with this type of measurement. In addition, some remarks are made with regard to how selection of patients with this methodology can be improved and how it may be used as a tool to evaluate the duration of action of antihypertensive agents. Finally, some potential disadvantages of ABPM as compared to conventional clinic blood pressure measurements are discussed.
\end{abstract}

Keywords: Ambulatory blood pressure monitoring; Hypertension; Antihypertensive agents; Clinical trials; Sample size; Placebo response

\section{Introduction}

Although the place of ambulatory blood pressure monitoring (ABPM) for the management of the individual hypertensive patient has still to be decided, this methodology has obtained a definitive place in the assessment of the blood-pressure-lowering effect of antihypertensive drugs in clinical trials.

Compared to convential clinic blood pressure measurements ABPM appears to have a number of favourable aspects and relatively few disadvantages (Table 1).

\footnotetext{
${ }^{*}$ Tel. (010)-4633762; Fax (010)-4634531.
}

In this paper we will focus on the question of the extent to which ABPM is subject to a placebo response and how it may influence the sample size of the study population, whereas the other

Table 1

Advantages and diasadvantages of ambulatory blood pressure monitoring in clinical trials with antihypertensive drugs

\begin{tabular}{ll}
\hline Advantages & Disadvantages \\
\hline Reduction of placebo effect & $\begin{array}{c}\text { Inconvenience for } \\
\text { the patient } \\
\text { Uncontrolled conditions }\end{array}$ \\
$\begin{array}{l}\text { Reduction of sample size } \\
\text { Evaluation of duration of effect }\end{array}$ & \\
$\begin{array}{l}\text { Evaluation of effect on diurnal } \\
\text { rhythm of blood pressure }\end{array}$ \\
Improved patient selection
\end{tabular}


points summarized in Table 1 will be discussed only briefly.

\section{Evaluation of duration of effect}

For the evaluation of the duration of effect of an antihypertensive compound it is necessary to perform blood pressure measurements at repeated intervals. Obviously this can be done by means of convential clinic blood pressure measurements; however, this is a laborious procedure and in addition it has the disadvantage that measurements can only be performed in a clinical setting while the patient is at rest. With ABPM a large number of blood pressure measurcments can be taken over an extended period of time with the patient active at home or at work. This has the advantage that information about the duration of the blood-pressure-lowering effect can be obtained under more real-life conditions.

As part of the evaluation of a new antihypertensive agent it is customary to compare the effect at the end of the dose-interval, the trough effect, with the maximal or peak antihypertensive effect. Calculation of the trough to peak ratio may be helpful by determining the dose interval of the antihypertensive agent. For example, for an antihypertensive agent with a claimed duration of action of $24 \mathrm{~h}$ the trough antihypertensive effect at the end of the dose interval should be at least $50 \%$ of the peak effect. In order to obtain approval for registration, the Food and Drug Aministration (FDA) in the United States requires information about the trough and peak effects of the antihypertensive agent under consideration [1].

\section{Evaluation of the effect on the diurnal rhythm of blood pressure}

In both normotensive and hypertensive subjects blood pressure shows a diurnal rhythm with a markedly lower blood pressure during the night than during the day. By means of ABPM it is possible to evaluate the effect of antihypertensive agents on this diurnal blood pressure rhythm.
Must of the classes of antihypertensive agents currently used have a similar effect on the day and night blood pressure, thereby leaving the normally occurring diurnal variation of blood pressure undisturbed.

The centrally acting sympathicolytic agent $\alpha$ methyldopa, which effectively lowers blood pressure during the day, has been shown to have little effect on nocturnal blood pressure [2] and similar observations have been made with the combined $\alpha$ - and $\beta$-adrenoceptor antagonist, labetolol [3]. From these observations it might be concluded that an increase in the activity of the sympathetic nervous system during the day contributes considerably to the higher level of blood pressure over this period.

The effect of antihypertensive agents on the diurnal blood pressure profile is not merely of academic interest, but may be important in the light of observations that a number of cardiovascular morbid events, such as myocardial infarction and ischaemia as well as thrombotic and haemorrhagic strokes, exhibit a diurnal rhythm as well [4-6].

\section{Selection of patients}

In almost all reported clinical trials with antihypertensive drugs convential blood pressure measurements have been used as an entry-criterion for the selection of patients. A consequence of this method of selection is that apart from subjects with genuine hypertension a number of subjects with so-called white-coat hypertension will be included as well. The effectiveness of antihypertensive agents in white-coat hypertensives is minimal or absent, as has been demonstrated for calcium entry blockers $[7,8]$. Inclusion of this category of subjects will therefore lead to a dilution of the antihypertensive effect of the agent studied.

Furthermore, administration of antihypertensive agents to subjects who are not truly hypertensive may be associated with more side-effects. It may be argued therefore that, instead of the convential clinic blood pressure measurements, $A B P M$ should be used as an entry-criterion to 
select patients for clinical trials with antihypertensive agents.

\section{Reduction of placebo effect}

According to Wolf a placebo response may be defined as any effect attributable to a pill, potion or procedure, but not its pharmacodynamic or specific properties [9]. The susceptibility of convential clinic blood pressure measurements to a placebo has been well established in some large intervention trials. One example is the Australian Trial in Mild Hypertension [10]. Subjects were eligible to enter this trial if their diastolic blood pressure during a run-in period of 4 weeks varied between 95 and $109 \mathrm{mmHg}$ (average of 6 measurements). At the end of the run-in period 1408 subjects were randomized for placebo. After an observation period of 3 years it appeared that 669 of these subjects, i.e. almost $50 \%$, had a diastolic blood pressure below $95 \mathrm{mmHg}$.

The way in which placebo lowers blood pressure is not precisely known. Habituation to the environment, to the procedure of blood pressure measurement and to the doctor or nurse, who measures the blood pressure, is probably the most important factor. Regression to the mean may also play a role. For an antihypertensive drug trial subjects are selected on the basis of an elevated blood pressure. On mere statistical grounds it can be expected that in a proportion of these subjects this abnormality diminishes with time. The inherent wide variability of blood pressure, of which the clinic blood pressure measurement is only a momentary estimate, as well as the expectations of the patient or physician with respect to treatment are factors which may also contribute to the placebo response.

The effect of placebo on ABPM has been investigated in several studies (Table 2). In a relatively large number of patients the Rembler ${ }^{\circledR}$ monitor has been used for the measurement of blood pressure. The Rembler ${ }^{\circledR}$ monitor is a nonautomatic recorder. The cuff has to be inflated by the patient himself, so that only day-time values of blood pressure can be obtained. When the day-time blood pressure values of the ambulatory
Table 2

Studies comparing effect of placebo on clinic and ambulatory blood pressure measurements

\begin{tabular}{lll}
\hline $\begin{array}{l}\text { Refer- } \\
\text { ence no. }\end{array}$ & $\begin{array}{l}\text { No. of } \\
\text { patients }\end{array}$ & $\begin{array}{l}\text { Methodology of blood } \\
\text { pressure monitoring }\end{array}$ \\
\hline 11 & 20 & Invasive (Oxford technique) \\
12 & 66 & Non-invasive (Rembler ${ }^{\circledR}$ monitor) \\
13 & 42 & Non-invasive (Copal ${ }^{\circledR 3}$ monitor) \\
14 & 10 & Non-invasive (Spacelabs ${ }^{\circledR}$ monitor) \\
\hline
\end{tabular}

recordings performed in the studies summarized in Table 2 were compared with untreated clinic blood pressures it appeared that the latter measurements were considerably higher (Fig. 1). The responses of clinic and ambulatory blood pressure measurements to placebo, given for 3-6 weeks, are depicted in Fig. 2. In response to placebo the decrements in clinic systolic and diastolic blood pressure were respectively 9.0 and 4.5 $\mathrm{mmHg}$. For the ambulatory measurements these decrements were considerably smaller: respectively $3.0 \mathrm{mmHg}$ systolic and $1.8 \mathrm{mmHg}$ diastolic. The small effect of placebo on ABPM is mainly present during the initial hours of the 24-h recording as has been shown by Mutti et al. [15].

On the basis of the above-mentioned data it can be concluded that ABPM, at least in short-

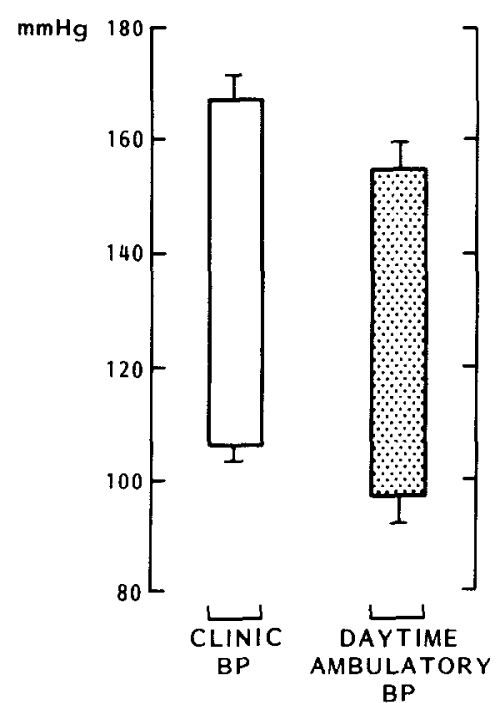

Fig. 1. Untreated clinic and daytime ambulatory blood pressures in 130 patients. Data are derived from the studies mentioned in Table 2. 


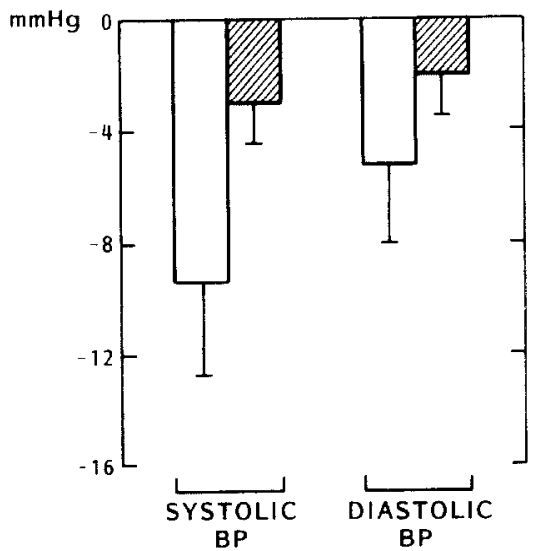

Fig. 2. Responses of clinic and daytime ambulatory systolic and diastolic blood pressure to placebo in 130 patients. Open bars = clinic blood pressure; hatched bars = daytime ambulatory blood pressure.

term studies, is only minimally affected by placebo. This almost absent effect of placebo on ABPM provides strong evidence that the placebo effect on clinic blood pressure is mainly caused by a decrease of the so-called white-coat effect or pressor response and not by a real sustained reduction of blood pressure.

\section{Effects on sample size of the study}

When the effectiveness of antihypertensive therapy is investigated, it has to be taken into consideration that blood pressure is a highly variable parameter from one occasion to another. Hills and Armitage have outlined the requirements for estimating the sample size for a crossover or parallel group trial [16]. Three factors are predetermined by the investigator. These factors are: (1) the power or precision of the study (usually set at 80 or $90 \%$ ), the power being the probability of obtaining a significant result if there is a true difference between the treatments; (2) the level of significance acceptable (usually set at $p<0.05$ ); and (3) the minimum difference between treatments to be detected. Given these requirements, which are independent of the methodology of blood pressure measurement, the determinant of the sample size will be the variability or the rcpcatability of the blood pressurc measurement itself. It is clear that if the variability of the blood pressure measurement is high, it becomes more difficult to detect any effect of treatment and that a larger sample size has to be studied.

For blood pressure it appears that the repeatability can be markedly improved by increasing the number of measurements $[17,18]$. In a recent study we have measured blood pressure by convential standard mercury sphygmomanometry and with an oscillometric device for ABPM (Spacelabs 90207 blood pressure monitor) in 42 hypertensive patients on two occasions, 1 week apart, while the subjects were taking placebo. The clinic blood pressure measurements were performed in triplicate by an experienced investigator with the subject in a supine position for $20 \mathrm{~min}$. The three values obtained on each occasion were averaged. ABPM was measured 4 times per hour during the day and 2 times per hour during the night. From these recordings $24-\mathrm{h}$ averages of systolic and diastolic blood pressure were calculated. Between the first and second occasion the mean difference in clinic systolic and diastolic blood pressure was respectively 8.3 and $5.2 \mathrm{mmHg}$, with corresponding standard deviations of these differences of 13.4 and $7.3 \mathrm{mmHg}$. For 24-h ABPM the mean difference in systolic and diastolic blood pressure between the first and second occasion was respectively 2.3 and $2.2 \mathrm{mmHg}$, with corresponding standard deviations of 6.3 and $4.7 \mathrm{mmHg}$ (Fig. 3). Thus, compared to the clinic blood pressure measurements, the standard deviation of the differences (SDD) between the repeated 24-h ambulatory measurements was almost two-fold smaller. This reduced variability has important consequences for the number of patients to be studied in clinical trials. For a cross-over trial the number of patients to be studied can be calculated from the formula:

$n=10 \times S D D^{2} /$ Difference $^{2}$,

where $n=$ the number of patients, $S D D=$ standard deviation of differences between measure- 
ments and Difference $=$ predetermined treatment effect to be detected. Thus, for a similar treatment effect to be detected a two-fold reduction in variability means a four-fold reduction in the number of patients to be studied. Generally spoken, to halve the standard deviation of differences between trial readings will double the precision or power of the trial or allow a four-fold reduction in the number of patients to obtain an accurate result.

One remark has to be made. Although 24-h average blood pressures obtained by ABPM are
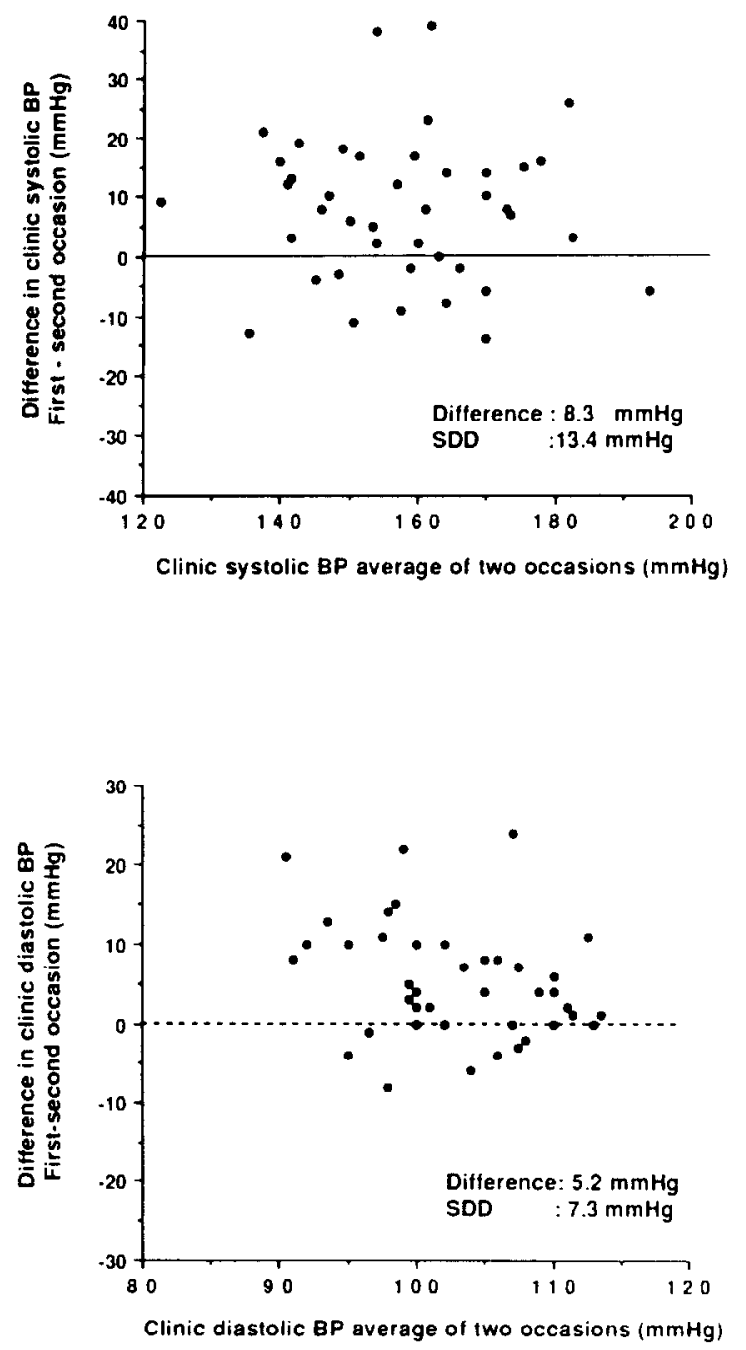

much more reproducible than clinic blood pressure measurements, the reproducibility of hourly blood pressure averages (mean of 2-4 values) obtained by ABPM appears to be quite variable. In a group of 15 subjects Trazzi ct al. reportcd that SDD values of hourly averages ranged from 7 to $18 \mathrm{mmHg}$ for systolic and from 6 to 13 $\mathrm{mmHg}$ for diastolic blood pressure [19]. Obviously, this limited hourly reproducibility has important consequences for sample size calculations if one is interested in the duration of action of antihypertensive drugs.
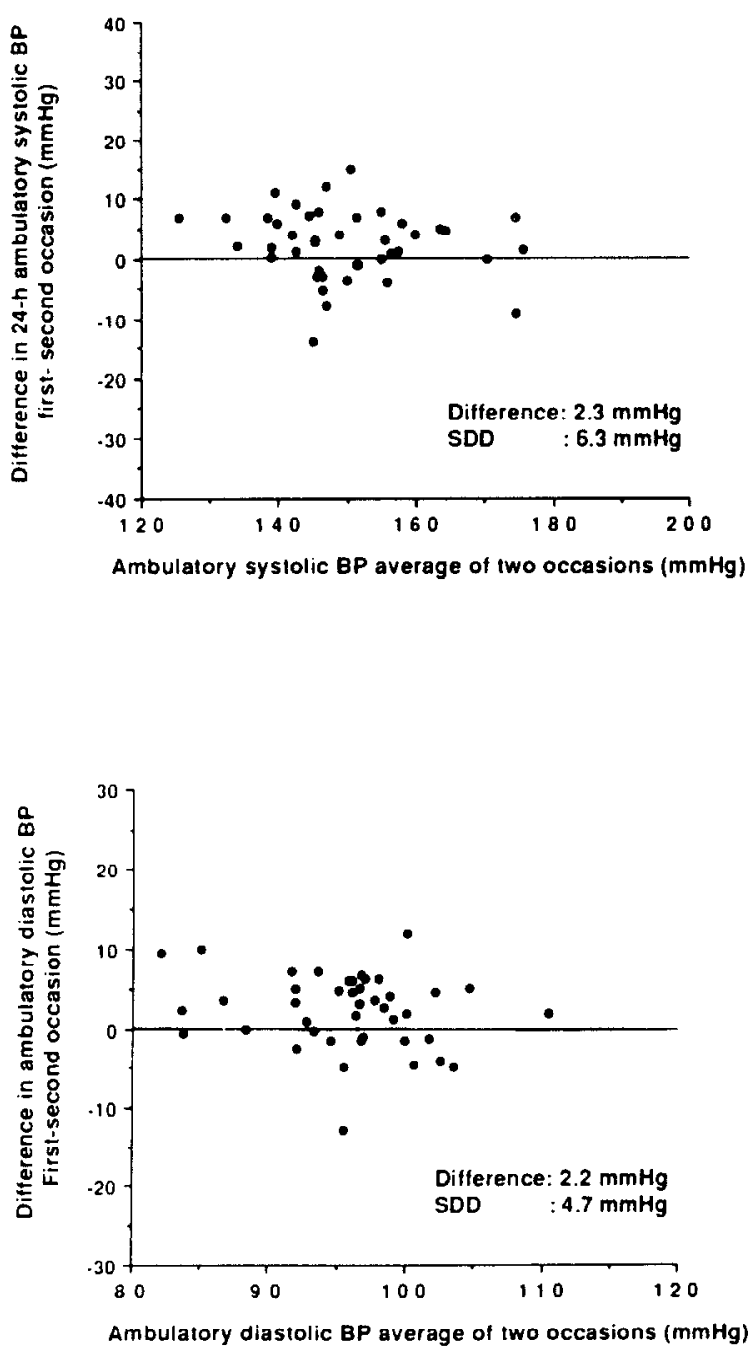

Fig. 3. Variability of repeated clinic and 24-h ambulatory pressure measurements. On both occasions all subjects were on placebo. The time interval between the two occasions was 1 week. SDD denotes standard deviation of differences. 


\section{Disadvantages of ambulatory blood pressure recording}

The most important disadvantage of ABPM is that the environmental conditions of blood pressure measurement as compared to clinic blood pressure measurements are less strictly standardized. Especially in a clinical trial all efforts should be made to standardize the conditions of measurements as much as possible. This can best be achieved by performing the measurements on similar days of the week and by introducing timed periods of controlled activity like walking, sitting, watching television etc. It is also important that on the occasions when blood pressure is measured, patients have fixed times for their nightrest and meals.

A second disadvantage of $A B P M$ is that the number of recordings that can be performed in one and the same patient is limited. Most patients do not feel very comfortable when they are equipped with blood pressure monitors that measure their blood pressure at frequent intervals both during the day and during the night. In a relatively large proportion of patients, ABPM has a negative influence on the quality of sleep. Whether this disturbance of sleep has an influence on the blood pressure level itself remains uncertain. Parati et al. found that the average overnight reduction in intra-arterial blood pressure during sleep was preserved during simultaneous recordings with an ambulatory blood pressure monitor [20]. In contrast, in a recent study Davies et al. found that blood pressure measurements during sleep with an ambulatory blood pressure monitor cause a variable rise in simultaneously recorded beat to beat systolic and diastolic blood pressure and, additionally, that these measurements are often associated with electroencephalographic arousal from sleep [21].

\section{Conclusion}

For the evaluation of the effectiveness and duration of action of antihypertensive drugs ABPM offers many advantages when compared with convential clinic blood pressure measure- ments. The almost complete elimination of the placebo response and the much better repeatability of ABPM allow smaller numbers of patients to be studied. This reduces costs and, more importantly, improves safety, because fewer patients have to be exposed to a new antihypertensive agent in the initial phase of clinical studies.

\section{References}

[1] Rose M, McMahon FG. Some problems with antihypertensive drug studies in the context of the new guidelines. Am J Hypertens 1990;3:151-155.

[2] Gould BA, Hornung RS, Kieso HA, Kashman PMM, Raftery EB. An intra-arterial profile of methyldopa. Clin Pharmacol Ther 1983;33:438-444.

[3] De Quattro V, De-Ping Lee D, Allen J, Sirgo M, Plachetka J. Labetolol blunts morning pressor surge in systolic hypertension. Hypertension 1988;11(Suppl 1):11981201.

[4] Muller JE, Stone PE, Turi ZG et al. Circadian variation in the onset of acute myocardial infarction. $\mathrm{N}$ Engl $\mathrm{J}$ Med 1985;313:315-322.

[5] Mulcahy D, Keegan J, Crean P et al. Silent myocardial ischemia in chronic stable angina: a study of its frequence and characteristics in 150 patients. $\mathrm{Br}$ Heart $J$ 1988;60:417-423.

[6] Tsementzis SA, Gill JS, Hitchcock ER, Gill SK, Beevers DG. Diurnal variation of and activity during the onset of stroke. Neurosurgery 1985;17:901-904.

[7] Weber MA, Cheung DG, Graettinger WF, Lipson JL. Characterization of antihyertensive therapy by whole-day blood pressure monitoring. J Am Med Assoc 1988;259:3281-3285.

[8] Lacourciere Y, Poirier I, Dion D, Provencher P. Antihypertensive effect of isradipine administered once or twice daily on ambulatory blood pressure. Am $\mathrm{J}$ Cardiol 1990;65:467-472.

[9] Wolf S. The pharmacology of placebo. Pharmacol Rev 1959;11:689-705.

[10] Doyle AE. Response to placebo treatment in hypertension. Hypertension 1983;5(Suppl III):3-4.

[11] Gould BA, Mann S, Davies AB, Altman DG, Raftery EB. Does placebo lower blood pressure? Lancet 1981;ii:1377-1381.

[12] Dupont AG, Van der Niepen P, Six RO. Placebo does not lower ambulatory blood pressure. Br J Clin Pharmacol 1987;24:106-109.

[13] Conway J, Johnston J, Coats A, Somers V, Sleight P. The use of ambulatory blood pressure monitoring to improve the accuracy and to reduce the numbers of subjects in clinical trials of antihypertensive agents. J Hypertens 1988:6:111-116. 
[14] Parati G, Pomidossi G, Casadei R, et al. Evaluation of the antihypertensive effect of celiprolol by ambulatory blood pressure monitoring. Am J Cardiol 1988;61:27C$33 \mathrm{C}$.

[15] Mutti E, Trazzi S, Omboni S, Parati G, Mancia G. Effect of placebo on 24-h non-invasive ambulatory blood pressure. J Hypertens 1991;9:361-364.

[16] Hills M, Armitage P. The two-period cross-over clinical trial. Br J Clin Pharmacol 1979;7:7-20.

[17] Conway J, Coats J. Value of ambulatory blood pressure monitoring in clinical pharmacology. J Hypertens 1989;7(Suppl 3);S29-S33.

[18] Trazzi S, Mutti E, Frattola A, Imholz B, Parati G, Mancia G. Reproducibility of non-invasive and intraarterial blood pressure monitoring: implications for stud- ies on antihypertensive treatment. J Hypertens 1991:9:115-119.

[19] Mancia G, Omboni S, Parati G, Trazzi S, Mutti E. Limited reproducibility of hourly blood pressure values obtained by ambulatory blood pressure monitoring: implications for studies on antihypertensive drugs. J Hypertens 1992;10:1531-1535.

[20] Parati G, Pomidossi G, Cassadei R, et al. Ambulatory blood pressure monitoring does not interfere with the haemodynamic effects of sleep. J Hypertens 1985;3(Suppl 2):107-109S.

[21] Davies RJO, Jenkins NE, Stradling JR. Effect of measuring ambulatory blood pressure on sleep and on blood pressure during sleep. Br Med J 1994;308:820-823. 